\title{
Heart rate response during 6-minute walking testing predicts outcome in operable chronic thromboembolic pulmonary hypertension
}

Manuel Jonas Richter ${ }^{1,2^{*}+}$, Katrin Milger ${ }^{2,3+}{ }^{\text {, Khodr Tello }}{ }^{2}$, Philipp Stille ${ }^{2}$, Werner Seeger ${ }^{2}$, Eckhard Mayer ${ }^{4}$, Hossein A. Ghofrani $i^{1,2,5}$ and Henning Gall ${ }^{2}$

\begin{abstract}
Background: Six-minute walk test (6MWT) is routinely performed in chronic thromboembolic pulmonary hypertension (CTEPH) before pulmonary endarterectomy (PEA). However, the clinical relevance of heart rate response $(\Delta \mathrm{HR})$ and exercise-induced oxygen desaturation (EID) during 6MWT is remaining unknown.

Methods: Patients undergoing PEA in our center between 03/2013-04/2014 were assessed prospectively with hemodynamic and exercise parameters prior to and 1 year post-PEA. Patients with symptomatic chronic thromboembolic disease (mean pulmonary artery pressure (mPAP) $<25 \mathrm{mmHg}$ ) and clinical relevant obstructive pulmonary disease were excluded. The following definitions were used: $\Delta H R=$ (peak HR - resting HR), percent heart rate reserve $(H R R)=\left(\right.$ peak HR -rest HR) $/\left(220\right.$ - age - rest HR) $\times 100$ and $E I D=\mathrm{SpO}_{2} \leq 88 \%$.

Results: Thirty-seven patients (of 116 patients screened) with mPAP of $43.2 \pm 8.7 \mathrm{mmHg}$, pulmonary vascular resistance (PVR) of $605.5 \pm 228.7 \mathrm{dyn} * \mathrm{~s} / \mathrm{cm}^{5}$, cardiac index (Cl) of $2.4 \pm 0.5 \mathrm{l} / \mathrm{min} / \mathrm{m}^{2}$ and a $6 \mathrm{MWT}$-distance of $404.7 \pm 148.4 \mathrm{~m}$ and a peak $\mathrm{VO}_{2}$ of $12.3 \pm 3.4 \mathrm{ml} / \mathrm{min} / \mathrm{kg}$ prior to PEA were included. Baseline $\Delta \mathrm{HR}$ during $6 \mathrm{MWT}$ was significantly associated with PVR 1 year post-PEA using linear regression analysis $(r=0.43, p=0.01)$. Multivariate analysis indicated an association of HRR during 6MWT and residual PH with a hazard ratio of 1.06 (95\% Confidence interval for hazard ratio $0.99-1.14, p=0.08$ ). EID was observed commonly during 6MWT but no correlations to outcome parameters were found.
\end{abstract}

Conclusions: This is the first prospective study to describe an association of $\Delta \mathrm{HR}$ during $6 \mathrm{MWT}$ with pulmonary hemodynamics 1 year post-PEA. Our preliminary results indicate that HRR derived from 6MWT is of clinical significance. EID was commonly observed, albeit failed as a significant prognostic factor.

Keywords: Oxygen desaturation, Heart rate response, Chronic thromboembolic pulmonary hypertension, Pulmonary endarterectomy

\footnotetext{
* Correspondence: manuel.richter@innere.med.uni-giessen.de

${ }^{\dagger}$ Equal contributors

${ }^{1}$ Department of Pneumology, Kerckhoff Heart, Rheuma and Thoracic Center,

Bad Nauheim, Germany

${ }^{2}$ Department of Internal Medicine, Justus-Liebig-University Giessen,

Universities of Giessen and Marburg Lung Center (UGMLC), Member of the

German Center for Lung Research (DZL), Giessen, Germany

Full list of author information is available at the end of the article
} 


\section{Background}

Chronic thromboembolic pulmonary hypertension (CTEPH) is defined by an elevation of mean pulmonary arterial pressure (mPAP) and pulmonary vascular resistance (PVR) caused by unresolved pulmonary vascular obstruction due to recurrent embolism [1, 2]. Mechanical obstruction in proximal parts of the pulmonary vascular system and secondary small-vessel arteriopathy in the non-obstructed areas are causes of disease progression and lead to extensive right ventricle (RV) dysfunction, loading and failure $[1,2]$. Pulmonary endarterectomy (PEA) is the gold standard in case of surgical accessible CTEPH and offers a potential curative treatment with an improved functional outcome and high survival rates $[3,4]$. Predictors of favorable outcome after PEA are important in daily clinical practice and include preoperative forced expiratory volume in $1 \mathrm{~s}$ (FEV1), heart-type fatty acid-binding protein (H-FABP) and cardiac index $(\mathrm{CI})[5,6]$. In addition, 6-minute walk testing (6MWT) with measurement of the distance covered (6MWD) is performed routinely before and after PEA as a tool to assess disease severity, functional capacity or long-term outcome [7, 8]. Moreover, preoperative 6MWD correlates with 3-month survival after PEA [9]. However, the prognostic relevance of additional parameters derived from the 6MWT were not evaluated in detail before. Presently, it is unknown whether exercise-induced oxygen desaturation (EID) or heart rate response $(\Delta \mathrm{HR})$ during 6MWD associate with disease severity, functional or hemodynamic outcome in operable CTEPH. Nevertheless, patients with $\mathrm{CTEPH}$ frequently display significant EID with inadequate increase in heart rate (HR) during exercise [10]. Previously, EID has been related to reduced exercise performance and increased mortality in patients with chronic obstructive pulmonary disease (COPD) and pulmonary fibrosis $[11,12]$, whereas $\triangle H R$ in pulmonary arterial hypertension (PAH) was analyzed in the context of baseline exercise capacity and functional improvements under targeted therapy [13]. Furthermore, chronotropic incompetence derived from heart rate reserve (HRR) was identified as an important and independent predictor of mortality in population based studies [14]. Moreover, parameters such as peak oxygen uptake $\left(\mathrm{VO}_{2}\right)$, peak systolic and diastolic blood pressure derived from cardio pulmonary exercise testing (CPET) are better correlated with functional class and prognosis than resting hemodynamic parameters in PAH [15]. So far, the significance of $\triangle H R$ and EID during 6MWD in comparison to CPET is remaining unknown in CTEPH.

We hypothesized that EID during 6MWD in CTEPH might be of prognostic relevance and related to the extent of dyspnea. Moreover, we speculated that the $\Delta \mathrm{HR}$ derived from the $6 \mathrm{MWT}$ reflects the inappropriate response of the RV with insufficient increase of the cardiac output (CO) to exercise, as it was shown for CPET [16]. Therefore, our aim was to prospectively investigate $\triangle \mathrm{HR}$ and EID during 6MWT in patients with operable CTEPH and their associations with clinical symptoms and hemodynamic outcome 1 year postPEA. In addition, we aim to compare $\triangle H R$ and EID assessed during 6MWT with CPET prior PEA in regard of their predictive significance.

\section{Methods \\ Patients}

All CTEPH patients undergoing PEA between March 2013 and April 2014 at the Department of Thoracic surgery, Kerckhoff-Clinic, Bad Nauheim, Germany were prospectively screened. After exclusion, 67 patients entered the study pre-PEA, while only patients with complete baseline and 1 year post-PEA hemodynamic data were analyzed ( $n=37)$ (Fig. 1$)$. Baseline and followup right heart catheter ( $\mathrm{RHC}$ ) were not mandatorily performed in-house, as the Kerckhoff-Clinic is a national referral center. Patients with symptomatic chronic thromboembolic disease (mean pulmonary artery pressure $<25 \mathrm{mmHg}$ at baseline [17]), obstructive pulmonary disease (forced expiratory volume in $1 \mathrm{~s} /$ vital capacity (FEV1/VC) $\leq 70 \%$ ) were excluded.

CTEPH was diagnosed according to current guidelines [18] and operability was assessed by a multidisciplinary board including pulmonary physicians, PEA surgeons and pulmonary radiologists. All patients received oral anticoagulants for at least 3 months and underwent PEA according to the protocol of the Kerckhoff-Clinic [19]. Residual PH 1 year post-PEA were defined by mPAP $>25 \mathrm{mmHg}$ and PVR $>240$ dyn*s $/ \mathrm{cm}^{5}$ at rest [20] while CTEPH type was classified by the surgical specimen as described previously [21].

All included patients gave written, informed consent, and the study was approved by the by the ethics committee of the Faculty of Medicine at the University of Giessen (Approval No. 67/14).

The following definitions were used: $\Delta H R=$ (peak HR resting $\mathrm{HR})$ [22], $\mathrm{HRR}=($ peak $\mathrm{HR}$-rest $\mathrm{HR}) /(220$ - age rest $\mathrm{HR}) \times 100$ [23] and EID = oxygen pulse saturation $\left(\mathrm{SpO}_{2}\right) \leq 88 \%[12,24]$.

\section{6-minute walk test}

All patients performed 6MWT at the Kerckhoff-Clinic according to current guidelines [25]. Patients were instructed to walk at their own pace while standard phrases were communicated [25]. HR and oxygen pulse saturation $\left(\mathrm{SpO}_{2}\right)$ using pulse oximetry $\left(\mathrm{Oximax}{ }^{\mathrm{TM}}, \mathrm{N}-65^{\mathrm{TM}}\right.$, Covidien AG, USA) were measured at baseline and every minute until minute 6 . In addition, patients were asked to quantify their sensation of dyspnea with the BORG scale [26]. As described previously, the respiratory therapist checked that 


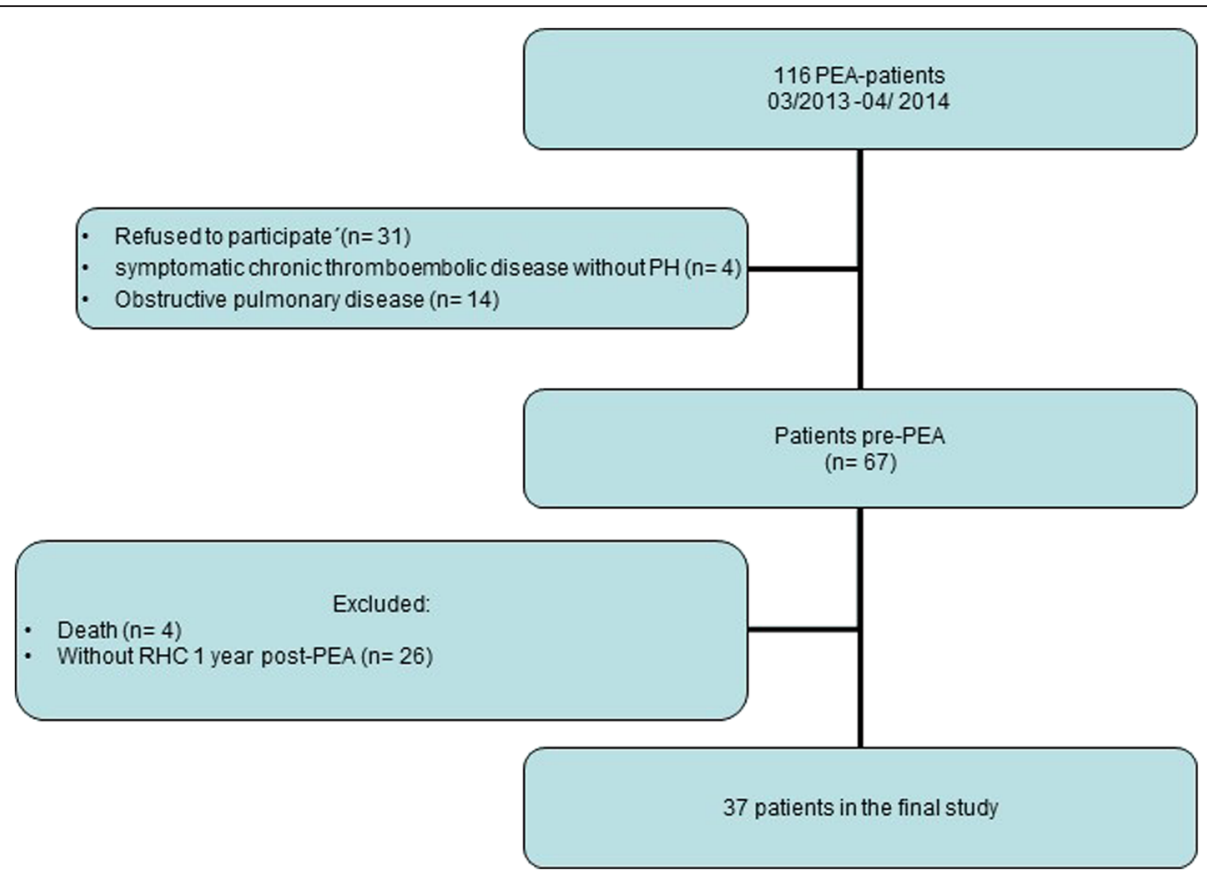

Fig. 1 Flow chart of patient selection. RHC: right heart catheterization, PEA: pulmonary endarterectomy, PH: pulmonary hypertension

the pulse oximeter had an acceptable signal before beginning all tests and carefully instructed the patient [12]. Patients already receiving supplemental oxygen at rest performed 6MWT with their current oxygen dosage. $6 \mathrm{MWD}$ was defined as the maximal achieved walk distance in $\mathrm{m}$ at room air or oxygen-supplemented.

\section{Cardio-pulmonary exercise testing}

Prior PEA patients performed a symptom-limited incremental CPET using a ramp protocol with an incremental rate of 5 to $15 \mathrm{~W} / \mathrm{min}$ judged by the operator (Masterscreen $\mathrm{CPX}^{\circ}$, Carefusion ${ }^{\circ}$ ), according to current recommendations [27]. Patients receiving supplemental oxygen and exhibited a resting $\mathrm{SpO}_{2}$ less than $88 \%$ were excluded. $\mathrm{Pa}-$ tients were asked to exercise up to their individual limit, while exercise was terminated due to exercise-limiting symptoms by the patient. Dead space ventilation $\left(\mathrm{V}_{\mathrm{D}} / \mathrm{V}_{\mathrm{T}}\right)$ was calculated using the Bohr- formula, while absolute dead space $\left(V_{D}\right)$ was assessed in milliliters [28].

\section{Baseline parameters}

Hemodynamic, laboratory, echocardiography data were collected before and after PEA in all included patients. Right heart echocardiography assessed pulmonary artery systolic pressure (PASP) and tricuspid annular plane systolic excursion (TAPSE) according to current recommendations [29].

\section{Statistical analysis}

Data are presented as mean \pm standard deviation (SD) for normally distributed parameters or median [interquartile range (IQR)]. As appropriate the 2-tailed $T$-test, paired $T$ test, Wilcoxon Signed Rank test, Mann-Whitney- $U$-test or Pearson Chi-Square test was used to test for differences between groups. Linear regression analysis was performed between follow-up hemodynamic parameters and baseline 6MWT and CPET characteristics. In addition, all baseline parameters were analyzed univariate in a logistic regression model with residual $\mathrm{PH}$ as dependent variable. Then all parameters of the univariate analysis with a $p$-value $<0.15$, were entered into a backward stepwise multivariate logistic regression model to predict residual PH 1 year post-PEA. $P$-value of $<0.10$ was considered statistically significant in the multivariate analyses. Statistical analysis was performed using SPSS, version 21.0 (IBM, Armonk, NY).

\section{Results}

\section{Baseline}

Thirty-seven CTEPH patients with a mean age of $61 \pm$ 12 years and a body mass index of $27.9 \pm 5.8 \mathrm{~kg} / \mathrm{m}^{2}$ mostly in WHO functional class III were included. Patients showed impaired pulmonary hemodynamics with a precapillary pattern, substantially reduced $\mathrm{CI}$ and elevated PVR before PEA. Concomitant right heart echocardiography showed an elevated PASP and a reduced TAPSE. Lung function testing revealed no significant obstructive or restrictive ventilatory abnormalities. During $6 \mathrm{MWT}$ peak $\mathrm{SpO}_{2}$ decreased to $88.8 \pm 5.6 \%$ with a $\Delta \mathrm{SpO}_{2}$ of $-5.1 \pm 4.4 \%$, HR increased to a mean peak of $117.1 \pm 18.8$ beats" $\mathrm{min}^{-1}$ while $\Delta \mathrm{HR}$ was $32.6 \pm 14.7$ 
Table 1 Baseline characteristics

\begin{tabular}{|c|c|c|c|}
\hline & Baseline & One year post-PEA & $p$-value \\
\hline Patients, $n$ & 37 & & \\
\hline Male/female & 20/17 & & \\
\hline Age (y) & $61 \pm 12$ & & \\
\hline $\mathrm{BMI}\left(\mathrm{kg} / \mathrm{m}^{2}\right)$ & $27.9 \pm 5.8$ & & \\
\hline WHO functional class, (\%) & & $b$ & 0.7 \\
\hline । & none & 29.6 & \\
\hline$\|$ & 27 & 48.1 & \\
\hline III & 62.2 & 22.2 & \\
\hline IV & 10.8 & None & \\
\hline \multicolumn{4}{|l|}{$6 \mathrm{MWT}$} \\
\hline $6 \mathrm{MWD}(\mathrm{m})$ & $404.7 \pm 148.4$ & $453.4 \pm 126.8$ & 0.1 \\
\hline Rest $\mathrm{SpO}_{2}(\%)$ & $93.9 \pm 2.7$ & $95.6 \pm 2.9$ & 0.043 \\
\hline Peak SpO2 (\%) & $88.8 \pm 5.6$ & $92.2 \pm 4.5$ & 0.003 \\
\hline$\Delta \mathrm{SpO} 2(\%)$ & $-5.1 \pm 4.4$ & $-3.5 \pm 4.1$ & 0.15 \\
\hline Rest HR (beats ${ }^{b}$ min-1) & $83.8 \pm 14.4$ & $83.5 \pm 13.9$ & 0.98 \\
\hline Peak HR (beats $\left.{ }^{\mathrm{b}} \mathrm{min}-1\right)$ & $117.1 \pm 18.8$ & $107.8 \pm 17.1$ & 0.041 \\
\hline$\Delta \mathrm{HR}\left(\right.$ beats $\left.^{\mathrm{b}} \min -1\right)$ & $32.6 \pm 14.7$ & $24.3 \pm 12.9$ & 0.038 \\
\hline $\operatorname{HRR}(\%)$ & $45.1 \pm 20.6$ & $33.4 \pm 16.7$ & 0.022 \\
\hline$\Delta$ Borg & $4.4 \pm 2.2$ & $3.1 \pm 2.4$ & 0.006 \\
\hline Supplemental Oxygen ( $n, \%)$ & $9(24.3)$ & $3(8.1)^{b}$ & 0.33 \\
\hline \multicolumn{4}{|l|}{ Lung function } \\
\hline FEV1 (\% pred.) & $88.7 \pm 13.8$ & $88.2 \pm 12.5$ & 0.76 \\
\hline FEV1/FVC (\% pred.) & $96 \pm 10.3$ & $90.6 \pm 12.1$ & 0.05 \\
\hline TLC (\% pred.) & $99 \pm 13.5$ & $104.8 \pm 16.8$ & 0.11 \\
\hline VC (\% pred.) & $91.6 \pm 13.7$ & $94 \pm 12.7$ & 0.34 \\
\hline \multicolumn{4}{|l|}{$\mathrm{RHC}$} \\
\hline mPAP (mm Hg) & $43.2 \pm 8.7$ & $28.9 \pm 10.1$ & 0.001 \\
\hline RAP $(\mathrm{mm} \mathrm{Hg})$ & $5.9 \pm 4.1$ & $7.2 \pm 4.3$ & 0.15 \\
\hline PVR (dyne $e^{b} / \mathrm{cm}^{5}$ ) & $605.5 \pm 228.7$ & $328.1 \pm 241.4$ & 0.001 \\
\hline $\mathrm{Cl}\left(1 / \mathrm{min} / \mathrm{m}^{2}\right)$ & $2.4 \pm 0.5$ & $2.7 \pm 1.3$ & 0.048 \\
\hline PAWP (mm Hg) & $9.5 \pm 4.6$ & $10.6 \pm 4.5$ & 0.19 \\
\hline \multicolumn{4}{|l|}{ Echocardiography } \\
\hline TAPSE (mm) & $17.2 \pm 4.3$ & $17.5 \pm 3.2$ & 0.62 \\
\hline PASP (mm Hg) & $69.8 \pm 25.1$ & $56.8 \pm 23.7$ & 0.041 \\
\hline \multicolumn{4}{|l|}{$\mathrm{CPET}^{\mathrm{C}}$} \\
\hline Peak $\mathrm{VO}_{2}(\mathrm{ml} / \mathrm{min} / \mathrm{kg})$ & $12.3 \pm 3.4$ & $14.2 \pm 4.2$ & 0.33 \\
\hline Rest $V_{D} N_{T}$ & $35.2 \pm 7.6$ & $32 \pm 5.8$ & 0.77 \\
\hline Peak $V_{D} N_{T}$ & $39.5 \pm 8.7$ & $35 \pm 11.9$ & 0.6 \\
\hline Rest $V_{D}, L$ & $0.35 \pm 0.17$ & $0.29 \pm 0.11$ & 0.39 \\
\hline Peak $V_{D}, L$ & $0.65 \pm 0.25$ & $0.72 \pm 0.21$ & 0.55 \\
\hline Rest $\mathrm{SpO}_{2}(\%)$ & $94.2 \pm 1.7$ & $95.1 \pm 1.4$ & 0.10 \\
\hline Peak SpO2 (\%) & $89.3 \pm 1.4$ & $90.7 \pm 4.8$ & 0.86 \\
\hline$\Delta \mathrm{SpO} 2(\%)$ & $-4.9 \pm 5.8$ & $-4.4 \pm 4.3$ & 0.69 \\
\hline Rest HR (beats $\left.{ }^{b} \min -1\right)$ & $72.8 \pm 12.2$ & $81.2 \pm 11.2$ & 0.15 \\
\hline
\end{tabular}


Table 1 Baseline characteristics (Continued)

\begin{tabular}{|c|c|c|c|}
\hline Peak HR (beats ${ }^{\mathrm{b}}$ min-1) & $120.7 \pm 21.3$ & $122.9 \pm 19.6$ & 0.25 \\
\hline$\Delta \mathrm{HR}$ (beats ${ }^{\mathrm{b}}$ min-1) & $47.9 \pm 19.4$ & $41.7 \pm 16.3$ & 0.72 \\
\hline HRR (\%) & $54.7 \pm 21.1$ & $53.7 \pm 20.2$ & 0.92 \\
\hline \multicolumn{4}{|l|}{ Co-morbidities, n (\%) } \\
\hline Hypertension & $24(64.9)$ & Unchanged & \\
\hline Coronary heart disease & $4(10.8)$ & Unchanged & \\
\hline Renal insuffiency & $4(10.8)$ & Unchanged & \\
\hline \multicolumn{4}{|l|}{ Jamieson-Type, \% } \\
\hline । & 31 & & \\
\hline$\|$ & 31 & & \\
\hline III & 38 & & \\
\hline \multicolumn{4}{|l|}{ Laboratory } \\
\hline NT-proBNP (pg/ml) & $488.2[1004]$ & $245.0[422]^{\mathrm{a}}$ & 0.006 \\
\hline
\end{tabular}

beats" min $^{-1}$ and HRR was $45.1 \pm 20.6 \%$. Sensation of dyspnea as assessed by the BORG score showed a substantial increase ( $\triangle$ BORG $4.4 \pm 2.2$ ). In total, 9 patients underwent the $6 \mathrm{MWT}$ with supplemental oxygen (Table 1). In total, 34 patients performed CPET displaying an impaired exercise capacity while $V_{D}$ and $V_{D} / V_{T}$ exhibited a significant increase during exercise. During CPET peak $\mathrm{SpO}_{2}$ decreased to $89.3 \pm 1.4 \%$ with a $\Delta \mathrm{SpO}_{2}$ of $-4.9 \pm 5.8 \%$,

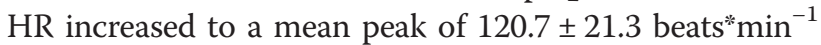
while $\Delta \mathrm{HR}$ was $47.9 \pm 19.4$ beats* $\mathrm{min}^{-1}$ and HRR was $54.7 \pm 22.1 \%$.

\section{One year post-PEA}

Hemodynamic and functional parameters significantly improved 1 year post-PEA as compared to baseline. Patients presented mostly in WHO functional class I and II, resting and peak $\mathrm{SpO}_{2}$ were significantly higher during 6MWT and peak HR, $\triangle \mathrm{HR}$ and HRR and NTproBNP were significantly lower 1 year post-PEA. A trend towards decrease was observed in resting $V_{D} / V_{T}$ and $\mathrm{V}_{\mathrm{D}}$ during CPET (Table 1). In total, 21 patients presented with residual $\mathrm{PH}$ displaying significantly elevated mPAP, PVR, NT-proBNP and reduced CI in comparison with non-residual $\mathrm{PH}$. In addition, patients with residual $\mathrm{PH}$ presented to a higher degree in WHO functional class III and showed, in comparison with non-residual $\mathrm{PH}$, a significant lower rest and peak $\mathrm{SpO}_{2}$ during 6MWT. HRR was significantly higher in patients with residual $\mathrm{PH}$ while $\triangle \mathrm{HR}$ showed a trend to higher values. Dyspnea as measured by BORG score was significantly increased in patients with residual $\mathrm{PH}$, while the administration of supplemental oxygen did not differ between groups (Table 2). In comparison with non-residual $\mathrm{PH}$ no significant differences of peak $\mathrm{VO}_{2}, \mathrm{SpO}_{2}, \mathrm{HR}$ or HHR derived from CPET were shown.

\section{Clinical relevance of exercise-induced oxygen desaturation}

Baseline characteristics were analyzed according to the presence of EID during 6MWT, while pulmonary hemodynamics, exercise capacity, $\mathrm{V}_{\mathrm{D}} / \mathrm{V}_{\mathrm{T}}$ and JamiesonType showed no significant differences in comparison. Interestingly, 12 out of 37 patients exhibited EID with a $\Delta \mathrm{SpO}_{2}$ of $10 \pm 1.1 \%$ (Table 3 ), while a rapid continuous desaturation was evident already starting at minute one of the 6MWT (Fig. 2a). BORG score at minute 3 was significantly higher in patients with EID, albeit $\triangle B O R G$ and peak BORG score showed a trend to higher values in EID (Fig. 2b).

There were no significant associations between rest $\mathrm{SpO}_{2}$, peak $\mathrm{SpO}_{2}$ or $\Delta \mathrm{SpO}_{2}$ with hemodynamic or functional parameters 1 year post-PEA during $6 \mathrm{MWT}$ and CPET (data not shown).

Univariate logistic regression analysis revealed that baseline peak $\mathrm{SpO}_{2}$ or $\Delta \mathrm{SpO}_{2}$ during $6 \mathrm{MWT}$ were not associated with the presence of residual $\mathrm{PH}$. Univariate analysis related rest $\mathrm{SpO}_{2}$ during $6 \mathrm{MWT}$ to residual $\mathrm{PH}$ with a hazard ratio of 0.8 (95\% Confidence interval for hazard ratio $0.6-1.06, p=0.11)$. However, additional stepwise backward multivariate analysis showed that rest $\mathrm{SpO}_{2}$ derived from 6MWT was not independently associated with the hemodynamic outcome 1 year post-PEA. In addition, rest $\mathrm{SpO}_{2}$, peak $\mathrm{SpO}_{2}$ or $\Delta \mathrm{SpO}_{2}$ derived from CPET failed as prognostic markers using univariate analysis (Table 4). 
Table 2 Parameters 1 year post-PEA according to non-residual and residual $\mathrm{PH}$

\begin{tabular}{|c|c|c|c|}
\hline & $\begin{array}{l}\text { Non-residual } \\
\mathrm{PH}\end{array}$ & Residual PH & $\begin{array}{l}p- \\
\text { value }\end{array}$ \\
\hline Patients, $n(\%)$ & $16(43.2)$ & $21(56.8)$ & \\
\hline WHO functional class, (\%) & & a & 0.002 \\
\hline । & 46.2 & None & \\
\hline$\|$ & 53.8 & 57.1 & \\
\hline III & None & 42.9 & \\
\hline IV & None & None & \\
\hline \multicolumn{4}{|l|}{$6 \mathrm{MWT}$} \\
\hline $6 \mathrm{MWD}(\mathrm{m})$ & $487.6 \pm 72.1$ & $432.4 \pm 148.9$ & 0.26 \\
\hline Rest $\mathrm{SpO}_{2}(\%)$ & $97 \pm 1.9$ & $94.8 \pm 3.1$ & 0.05 \\
\hline Peak SpO2 (\%) & $94.3 \pm 4.4$ & $90.8 \pm 4.1$ & 0.05 \\
\hline$\Delta \mathrm{SpO} 2(\%)$ & $-2.7 \pm 3.8$ & $-3.9 \pm 4.4$ & 0.47 \\
\hline Rest HR (beats ${ }^{\mathrm{a}} \mathrm{min}-1$ ) & $83.9 \pm 15.1$ & $83.2 \pm 13.9$ & 0.9 \\
\hline Peak HR (beats ${ }^{\mathrm{a}} \mathrm{min}-1$ ) & $101.6 \pm 15.2$ & $111.5 \pm 17.6$ & 0.2 \\
\hline$\Delta \mathrm{HR}$ (beats $\left.{ }^{\mathrm{a}} \mathrm{min}-1\right)$ & $17.8 \pm 6.3$ & $28.3 \pm 14.4$ & 0.06 \\
\hline HRR (\%) & $24.3 \pm 11.5$ & $28.3 \pm 14.4$ & 0.05 \\
\hline$\Delta$ Borg & $1.7 \pm 1.9$ & $3.9 \pm 2.4$ & 0.02 \\
\hline $\begin{array}{l}\text { Supplemental Oxygen ( } n \text {, } \\
\%)\end{array}$ & $1(6.3)$ & $2(9.5)$ & - \\
\hline \multicolumn{4}{|l|}{ Lung function } \\
\hline FEV1 (\% pred.) & $93.9 \pm 12.8$ & $83.9 \pm 10.6$ & 0.02 \\
\hline FEV1/FVC (\% pred.) & $95.8 \pm 13.9$ & $86.7 \pm 9.2$ & 0.03 \\
\hline TLC (\% pred.) & $104.2 \pm 15.2$ & $105.2 \pm 18.3$ & 0.87 \\
\hline VC (\% pred.) & $96.8 \pm 14.0$ & $92.1 \pm 11.6$ & 0.3 \\
\hline \multicolumn{4}{|l|}{ RHC } \\
\hline mPAP (mm Hg) & $19.7 \pm 3.2$ & $36 \pm 7.5$ & 0.001 \\
\hline RAP (mm Hg) & $5.5 \pm 2.4$ & $8.7 \pm 5.1$ & 0.04 \\
\hline PVR (dyne $\left.e^{\mathrm{a}} \mathrm{s} / \mathrm{cm}^{5}\right)$ & $218.3 \pm 280.9$ & $415.9 \pm 163.7$ & 0.012 \\
\hline $\mathrm{Cl}\left(1 / \mathrm{min} / \mathrm{m}^{2}\right)$ & $2.9 \pm 0.4$ & $2.6 \pm 0.6$ & 0.09 \\
\hline PAWP (mm Hg) & $8.9 \pm 3.8$ & $12.2 \pm 4.7$ & 0.037 \\
\hline \multicolumn{4}{|l|}{ Echocardiography } \\
\hline TAPSE (mm) & $18.3 \pm 2.7$ & $16.7 \pm 3.6$ & 0.2 \\
\hline PASP (mm Hg) & $50.6 \pm 29.2$ & $59.5 \pm 21.9$ & 0.5 \\
\hline \multicolumn{4}{|l|}{$\mathrm{CPET}^{\mathrm{b}}$} \\
\hline Peak $\mathrm{VO}_{2}(\mathrm{ml} / \mathrm{min} / \mathrm{kg})$ & $15.2 \pm 4.5$ & $13.3 \pm 3.9$ & 0.36 \\
\hline Rest $V_{D} N_{T}$ & $30.3 \pm 5.1$ & $35.3 \pm 6.4$ & 0.35 \\
\hline Peak $V_{D} N_{T}$ & $27.7 \pm 6.1$ & $41.2 \pm 12.1$ & 0.13 \\
\hline Rest $V_{D}, L$ & $0.22 \pm 0.07$ & $0.37 \pm 0.09$ & 0.09 \\
\hline Peak $V_{D}, L$ & $0.63 \pm 0.1$ & $0.78 \pm 0.26$ & 0.39 \\
\hline Rest $\mathrm{SpO}_{2}(\%)$ & $95.1 \pm 1.3$ & $93.7 \pm 1.8$ & 0.10 \\
\hline Peak SpO2 (\%) & $89.1 \pm 7.3$ & $89.6 \pm 4.6$ & 0.86 \\
\hline$\Delta \mathrm{SpO} 2(\%)$ & $-5.2 \pm 5.4$ & $-3.6 \pm 3.3$ & 0.69 \\
\hline Rest HR (beats ${ }^{a}$ min-1) & $76.1 \pm 12.4$ & $69.9 \pm 11.7$ & 0.15 \\
\hline Peak HR (beats ${ }^{a}$ min-1) & $125.3 \pm 22.4$ & $116.6 \pm 19.9$ & 0.25 \\
\hline
\end{tabular}

Table 2 Parameters 1 year post-PEA according to non-residual and residual $\mathrm{PH}$ (Continued)

\begin{tabular}{llll}
\hline$\Delta$ HR (beats $\left.{ }^{\mathrm{a}} \mathrm{min}-1\right)$ & $49.2 \pm 19.3$ & $46.7 \pm 19.9$ & 0.72 \\
HRR (\%) & $55.1 \pm 21.1$ & $54.3 \pm 21.8$ & 0.92 \\
Jamieson-Type, (\%) & & $\mathrm{a}$ & 0.15 \\
I & 14.2 & 46.7 & \\
II & 42.9 & 20 & \\
III & 42.9 & 33.3 & \\
Laboratory & & & \\
NT-proBNP (pg/ml) & $175.0[56-259]$ & $389.0[95-$ & 0.18 \\
& & $703]$ & \\
\hline
\end{tabular}

Values represent mean \pm SD or median [IQR]. ${ }^{a}=$ Pearson Chi-Square test,

${ }^{b} n=34$. For abbreviations see Table 1

\section{Clinical relevance of heart rate response}

During 6MWT an initial steep and then flattened HR response was observed (Fig. 2a). Baseline $\Delta H R$ during 6MWT was significantly associated with PVR 1 year postPEA ( $r=0.43, p=0.01)$ (Fig. 3a). However, using linear regression analysis $\triangle \mathrm{HR}$ showed no significant associations with mPAP (Fig. $3 \mathrm{~b}$ ) or other pulmonary hemodynamic parameters 1 year post-PEA (data not shown). There were no significant associations of rest HR, peak HR or HRR with hemodynamic parameters 1 year post-PEA (data not shown). Interestingly, HR parameters derived from CPET were not significantly associated with hemodynamic parameters 1 year post-PEA (data not shown).

Univariate logistic regression analysis revealed that baseline $\triangle \mathrm{HR}$ and HRR during 6MWT were significantly associated with the presence of residual $\mathrm{PH}$. The stepwise backward multivariate model associated HRR and Jamieson-Type with the hemodynamic outcome 1 year post-PEA. Intriguingly, no such association were found for the $\triangle \mathrm{HR}$ or HRR during CPET (Table 4).

\section{Discussion}

In the current study we could show for the first time that additional characteristics derived from 6MWT in operable CTEPH were of clinical relevance. The novel findings of the present study include 1) that EID during 6MWT is commonly observed in operable CTEPH prePEA, and 2) baseline HRR, but not EID, during 6MWT is associated with the hemodynamic outcome 1 year post-PEA. To the best of our knowledge, this is the first study that evaluated specific characteristics from 6MWT in a selected cohort of operable CTEPH patients and demonstrated their impact on the hemodynamic outcome post-PEA.

CTEPH patients with reduced exercise capacity, mostly in WHO functional class III and severely impaired pulmonary hemodynamics prior PEA were prospectively included. Our cohort demonstrated the typical precapillary $\mathrm{PH}$ pattern with significant impairment of RV function resulting in 
Table 3 Parameters at baseline according to exercise-induced oxygen desaturation during 6MWT

\begin{tabular}{|c|c|c|c|}
\hline & \multicolumn{2}{|l|}{ Baseline } & \multirow[b]{2}{*}{$p$-value } \\
\hline & EID & Non-EID & \\
\hline Patients, n (\%) & $12(32.4)$ & $25(67.6)$ & \\
\hline WHO functional class, (\%) & a & & 0.09 \\
\hline$\|$ & 33.3 & 24 & \\
\hline III & 41.7 & 72 & \\
\hline IV & 25 & 4 & \\
\hline \multicolumn{4}{|l|}{ 6MWT } \\
\hline $6 \mathrm{MWD}(\mathrm{m})$ & $448.9 \pm 206.8$ & $383.6 \pm 109.5$ & 0.22 \\
\hline Rest $\mathrm{SpO}_{2}(\%)$ & $92.0 \pm 2.2$ & $94.8 \pm 2.1$ & 0.002 \\
\hline Peak $\mathrm{SpO}_{2}(\%)$ & $81.9 \pm 4.4$ & $92.1 \pm 2.3$ & 0.001 \\
\hline$\Delta \mathrm{SpO} 2(\%)$ & $10.0 \pm 1.1$ & $2.7 \pm 0.4$ & 0.02 \\
\hline Rest HR (beats ${ }^{a}$ min-1) & $83.5 \pm 16.4$ & $84 \pm 13.7$ & 0.9 \\
\hline Peak HR (beats ${ }^{a}$ min-1) & $120.6 \pm 20.7$ & $115.5 \pm 18.1$ & 0.45 \\
\hline$\Delta \mathrm{HR}$ (beats $\left.{ }^{\mathrm{a}} \mathrm{min}-1\right)$ & $30.5 \pm 5.1$ & $30.4 \pm 2.4$ & 0.82 \\
\hline HRR (\%) & $49.5 \pm 8.1$ & $42.5 \pm 4$ & 0.53 \\
\hline$\Delta$ Borg & $4.7 \pm 0.7$ & $4.3 \pm 0.5$ & 0.52 \\
\hline Supplemental Oxygen $(n, \%)$ & $4(33.3)$ & $5(20)^{a}$ & 0.38 \\
\hline \multicolumn{4}{|l|}{ Lung function } \\
\hline FEV1 (\% pred.) & $83.6 \pm 11.9$ & $90.9 \pm 14.1$ & 0.15 \\
\hline FEV1/FVC (\% pred.) & $98.6 \pm 10.2$ & $94.8 \pm 10.3$ & 0.31 \\
\hline TLC (\% pred.) & $94.8 \pm 16.3$ & $100.8 \pm 12.1$ & 0.24 \\
\hline VC (\% pred.) & $85.8 \pm 10.3$ & $94.1 \pm 14.4$ & 0.1 \\
\hline \multicolumn{4}{|l|}{$\mathrm{RHC}$} \\
\hline mPAP (mm Hg) & $43.8 \pm 8.2$ & $42.9 \pm 9.0$ & 0.77 \\
\hline RAP (mm Hg) & $4.1 \pm 2.7$ & $6.6 \pm 4.4$ & 0.8 \\
\hline PVR (dyne $e^{a} / \mathrm{cm}^{5}$ ) & $640.1 \pm 231.0$ & $587.4 \pm 230.5$ & 0.53 \\
\hline $\mathrm{Cl}\left(1 / \mathrm{min} / \mathrm{m}^{2}\right)$ & $2.5 \pm 0.6$ & $2.4 \pm 0.5$ & 0.7 \\
\hline PAWP (mm Hg) & $9.3 \pm 2.8$ & $9.7 \pm 5.4$ & 0.8 \\
\hline \multicolumn{4}{|l|}{ Echocardiography } \\
\hline TAPSE (mm) & $16.6 \pm 4.3$ & $17.4 \pm 4.3$ & 0.63 \\
\hline PASP (mm Hg) & $77.2 \pm 30.7$ & $66.2 \pm 21.7$ & 0.24 \\
\hline \multicolumn{4}{|l|}{$\mathrm{CPET}^{\mathrm{C}}$} \\
\hline Peak VO $(\mathrm{ml} / \mathrm{min} / \mathrm{kg})$ & $13.1 \pm 1.3$ & $12.1 \pm 0.6$ & 0.65 \\
\hline Rest $V_{D} N_{T}$ & $33.0 \pm 7.3$ & $36.0 \pm 8$ & 0.48 \\
\hline Peak $V_{D} N_{T}$ & $39.0 \pm 12.0$ & $40.0 \pm 7.3$ & 0.78 \\
\hline Rest $V_{D}, L$ & $0.41 \pm 0.22$ & $0.34 \pm 0.14$ & 0.4 \\
\hline Peak $V_{D}, L$ & $0.67 \pm 0.33$ & $0.65 \pm 0.23$ & 0.9 \\
\hline Jamieson-Type, (\%) & a & & 0.72 \\
\hline I & 25 & 32 & \\
\hline$\|$ & 50 & 26 & \\
\hline III & 25 & 42 & \\
\hline \multicolumn{4}{|l|}{ Laboratory } \\
\hline NT-proBNP (pg/ml) & $414.0[1260.2]$ & $836.7[1521]^{\mathrm{b}}$ & 0.71 \\
\hline
\end{tabular}



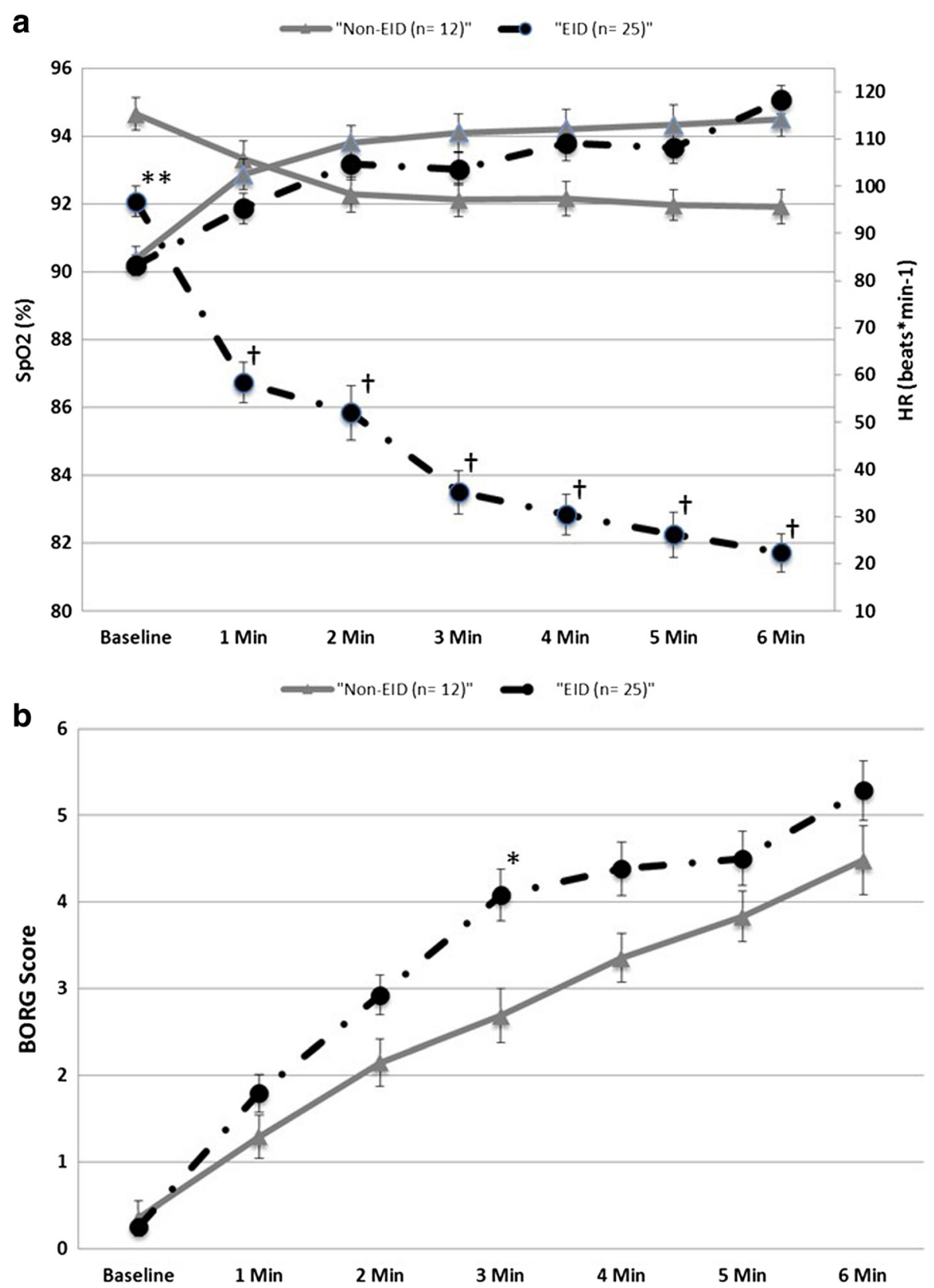

Fig. 2 a Heart rate (HR) and $\mathrm{SpO}_{2}$ during $6 \mathrm{MWT}$ according to exercise-induced oxygen desaturation (EID). Data are presented as mean \pm standard error of means. ${ }^{* *} p=0.002,{ }^{\dagger} p<0.001$ versus non-EID. (black line $=E I D ;$ grey line $=$ non-EID; $\boldsymbol{\Delta}=\mathrm{SpO}$; $\bullet=\mathrm{HR}$ ). $\mathbf{b}$ BORG score during $6 \mathrm{MWT}$ according to exercise-induced oxygen desaturation (EID). Data are presented as mean \pm standard error of means. ${ }^{*} p=0.02$ versus non-EID. (black line = EID; grey line = non-EID)

a reduced CI, elevated PVR and mPAP [7, 30-33]. Hemodynamic parameters and exercise capacity substantially improved 1 year post-PEA, albeit 21 patients (56.8 \%) presented with a residual $\mathrm{PH}$. In total, a significant improvement of the functional outcome 1 year post-PEA was observed as described previously [3, 4]. However, the high rate of residual $\mathrm{PH}$ in our selected cohort underlined that the individual outcome post-
PEA differed while rates of residual $\mathrm{PH}$ up to $35 \%$ were previously reported [20, 32, 34].

Our results describe for the first time that after exclusion of obstructive pulmonary diseases, almost $\sim 32 \%$ of operable CTEPH exhibited a substantial EID during 6MWT prior to PEA. Further, these patients reported an enhanced, albeit not significant, increased sensation of dyspnea during 6MWT. Our data support the high prevalence of EID 
Table 4 Baseline parameters as predictors of residual PH 1 year post PEA

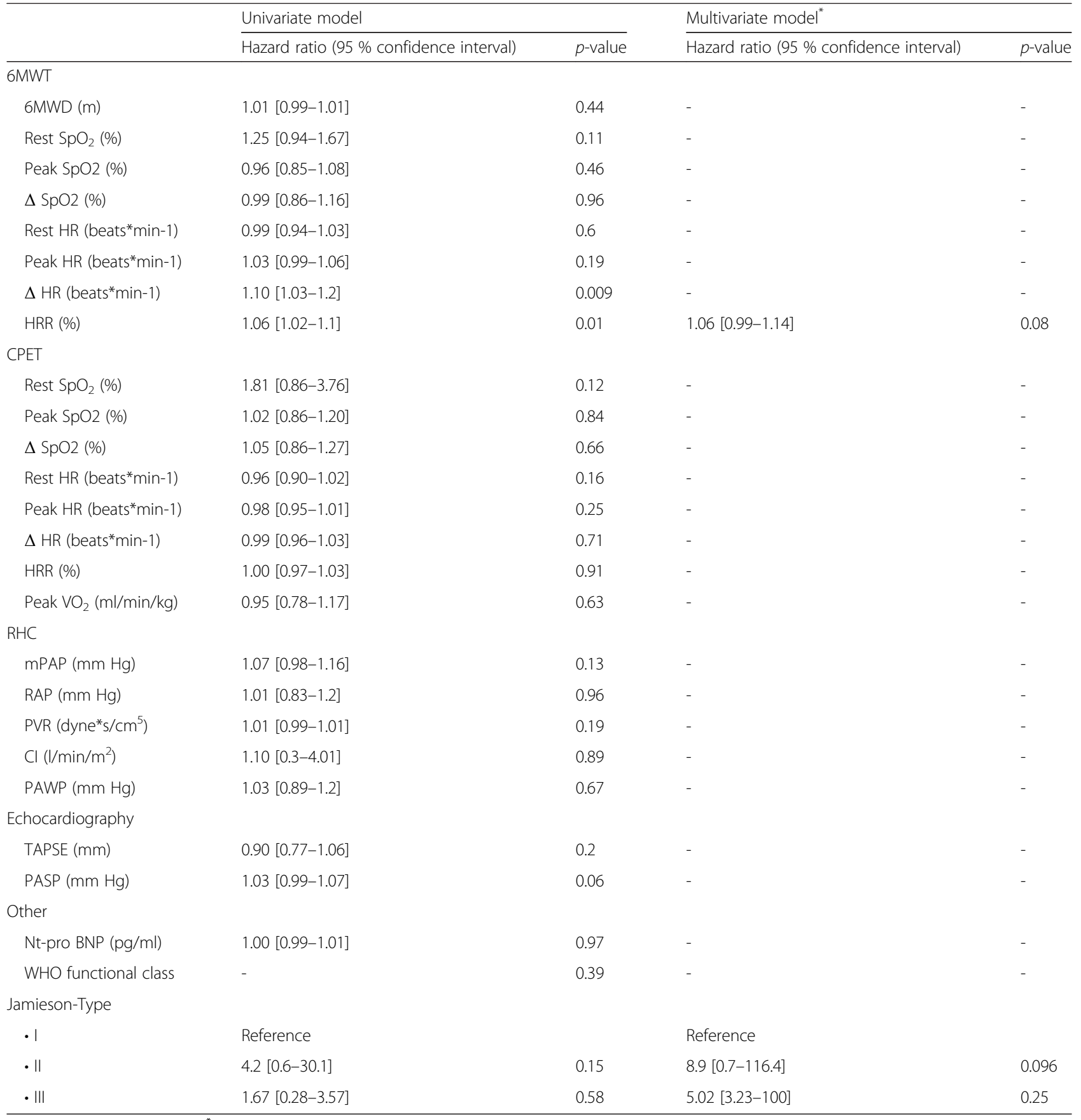

For abbreviations see Table 1. ${ }^{*}:$ backward stepwise logistic regression including variables with a $p$-value $<0.15$ in the univariate model

during 6MWT in CTEPH as described previously in a heterogeneous group of $\mathrm{PH}$ patients [35]. Furthermore, patients with residual PH showed significantly higher BORG scores and lower peak $\mathrm{SpO}_{2}$-levels 1 year post-PEA. The cause of EID and sensation of dyspnea is multifactorial in $\mathrm{CTEPH}$, including gas exchange abnormalities with exertional hypoxaemia due to increased $\mathrm{V}_{\mathrm{D}} / \mathrm{V}_{\mathrm{T}}$ and $\mathrm{V} / \mathrm{Q}$ mismatch, increased chemosensitivity as a stimulus of exercise hyperventilation and insufficient enhancement of $\mathrm{CO}$ due to RV dysfunction $[16,28,36]$. One can speculate that ventilatory inefficiency with an increased $V_{D} / V_{T}$ during exercise due to increased V/Q mismatch results in EID, which however couldn't be extrapolated by our data as significant correlations between EID and $V_{D} / V_{T}$ were lacking. The absence of significant associations between EID and $\mathrm{V}_{\mathrm{D}} / \mathrm{V}_{\mathrm{T}}$ might be related to the relatively small increase in $\mathrm{V}_{\mathrm{D}} / \mathrm{V}_{\mathrm{T}}$ during exercise observed in our cohort, as previously peak $\mathrm{V}_{\mathrm{D}} / \mathrm{V}_{\mathrm{T}}$ up to 50 were reported [36]. In addition, 


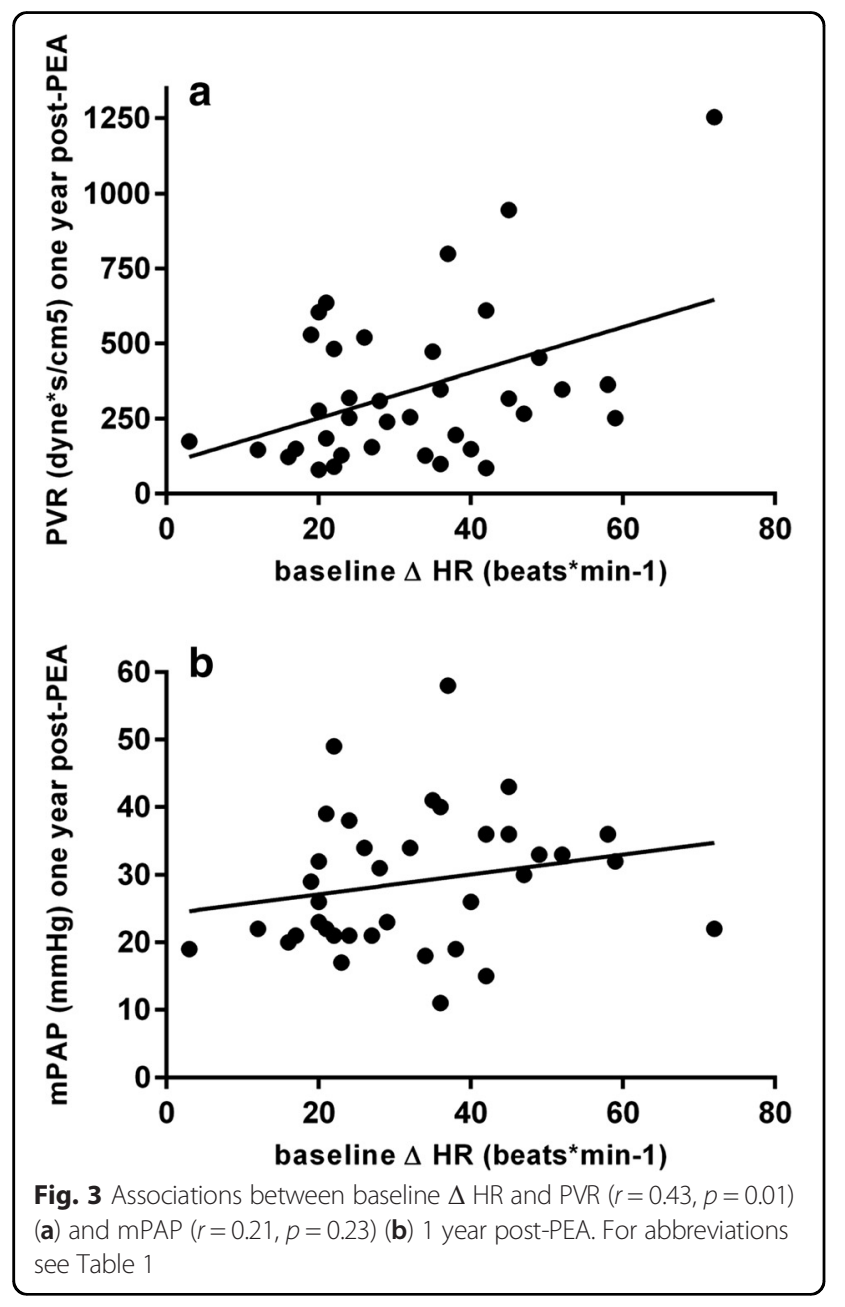

baseline resting $V_{D} / V_{T}$ was lower than previously reported indicating that pulmonary perfusion was, overall, slightly better in our cohort [30]. Surprisingly, baseline EID was neither associated with hemodynamic parameters 1 year post-PEA nor with the presence of residual $\mathrm{PH}$. Therefore our data emphasized that baseline EID failed as a surrogate marker to predict small-vessel arteriopathy, non-removable material or impaired RV reverse remodeling post-PEA which were identified as major causes of residual $\mathrm{PH}$ [20]. Nevertheless, EID was described as a powerful prognostic marker of overall survival in PAH, pulmonary fibrosis or COPD previously [11, 12, 37]. In addition, $\mathrm{SpO}_{2}$-levels or EID derived from CPET failed as relevant prognostic factors in our multivariate model.

Interestingly, our data indicated for the first time that $\triangle \mathrm{HR}$ prior PEA was related to PVR 1 year post-PEA in operable CTEPH patients. In addition, HRR during $6 \mathrm{MWT}$ was associated with the presence of residual $\mathrm{PH}$. Even though the association of an enhanced baseline HRR with the presence of residual PH might look paradoxical at first sight, its' pathophysiologic meaning in CTEPH is explicable. In general, exercising PAH patients exhibit a limited increase in stroke volume due to systolic and diastolic impairment, increased RV afterload and impaired ventriculoarterial coupling [38], while the diastolic pressure-volume relationship determines filling and $\mathrm{CO}$ [39]. In CTEPH the chronic obstructions in the pulmonary circulation lead to an increase in RV afterload and eventually to an impairment in RV function [40] such that the increase in $\mathrm{CO}$ is compensatory achieved through increases in HR. The disproportionate, albeit enhanced $\triangle H R$ at baseline inside a cohort with an overall impaired HRR, may reflect the severity of the disease. One can speculate that an enhanced HRR at baseline was mainly mirroring the inadequate response of the RV to adapt to higher load during exercise with a further impaired overall RV function and advanced RV remodeling. Therefore, HRR was associated with the hemodynamic outcome 1 year post-PEA in patients that were less prone to RV reverse remodeling. PEA immediately reverses the increased RV afterload in CTEPH patients, while the magnitude of reverse RV remodeling after PEA has been shown to correlate with changes in hemodynamics, restoration of ventriculoarterial coupling or reduction of RV systolic wall stress [33, 40-43]. However, it has been proposed that RV remodeling is only partly reversible because of diffuse myocardial fibrosis and differed individually [40]. As previously reported, regression of concentric hypertrophy and restoration of full right and left systolic function assessed by means of Cardiac Magnetic Resonance Imaging (cMRI) require a longer period of time of up to several years [43].

Interestingly, the $\triangle \mathrm{HR}$ and the HRR during CPET failed as prognostic marker in our cohort, highlighting the value of $6 \mathrm{MWT}$ in CTEPH. Being a self-paced submaximal effort test, it results in an aerobic steady-state, as opposed to CPET which is a maximal effort test. Thus differences in HRR with increased HR in more severely ill patients, as discussed above, may be observed in this test, but not at maximal effort. We therefore speculate that $\triangle \mathrm{HR}$ and the HRR assessed during 6MWT are superior in reflecting disease severity in a selected cohort of operable CTEPH patients. As shown previously, differences of the cardiac, ventilatory and metabolic response during 6MWT in comparison with CPET in patients with $\mathrm{PAH}$ are occurring $[15,44]$. CPET is associated with higher a minute ventilation, respiratory exchange ratio and maximal HR in comparison with 6MWT [44]. Therefore, Deboeck and coworkers concluded that 6MWT is a more realistic test for the determination of the exercise capacity than CPET and is more reflective of therapeutic interventions [44].

Limitations of the study are the small sample size and highly selected patient sample that excluded patients with obstructive pulmonary diseases. As we are a national referral center, the 1 year follow-up visit was not 
mandatorily performed in our center and accounts for the dropout rate of 26 patients. Furthermore, 31 patients refused participation at the time of screening due to various reasons. Taking together this drop out rate produces potential bias. In addition, since the rate of residual $\mathrm{PH}$ was higher than reported in the literature, a selection bias is possible. To confirm and elucidate the pathophysiological findings of our study, larger prospective studies including post-procedural cMRI and angiographies for quantification of reverse remodeling and residual perfusion impairments will be necessary.

\section{Conclusions}

This is the first prospective study to describe an association of additional characteristics derived from 6MWT with the hemodynamic outcome 1 year post-PEA in a selected cohort of operable CTEPH patients. Our preliminary results indicate that $\triangle \mathrm{HR}$ and HRR assessed during 6MWT are of clinical value in patients with operable CTEPH, and HRR during 6MWT may serve as a predictor of residual $\mathrm{PH}$. EID was commonly observed in operable CTEPH, albeit failed as a significant prognostic factor.

\section{Abbreviations}

6MWD: six-minute walking distance; 6MWT: six-minute walking test; Cl: cardiac index; CMRI: cardiac magnetic resonance imaging; COPD: chronic obstructive pulmonary disease; CPET: cardio-pulmonary exercise testing; CTEPH: chronic thromboembolic pulmonary hypertension; EID: exerciseinduced oxygen desaturation; FEV1: forced expiratory volume in $1 \mathrm{~s}$; FEV1/ VC: forced expiratory volume in $1 \mathrm{~s} / \mathrm{vital}$ capacity; FRC: functional residual capacity; H-FABP: heart-type fatty acid-binding protein; HR: heart rate: HRR: heart rate reserve; IQR: interquartile range; mPAP: mean pulmonary arterial pressure; NT-proBNP: N-terminal fragment of pro-brain natriuretic peptide; PAH: pulmonary arterial hypertension; PASP: pulmonary artery systolic pressure; PAWP: pulmonary arterial wedge pressure; PEA: pulmonary endarterectomy; PH: pulmonary hypertension; PVR: pulmonary vascular resistance; RAP: right atrial pressure; RHC: right heart catheter; RHC: right heart catheterization; RV: right ventricle; SD: standard deviation; $\mathrm{SpO}_{2}$ : oxygen pulse saturation; TAPSE: tricuspid annular plane systolic excursion; TLC: total lung capacity; VC: vital capacity; $V_{D}$ : absolute dead space; $V_{D} N_{T}$ : dead space ventilation; $\mathrm{VO}_{2}$ : oxygen uptake; WHO: World Health Organization; $\triangle \mathrm{HR}$ : heart rate response.

\section{Acknowledgements}

The manuscript is part of the doctoral thesis of Philipp Stille. Parts of the study results have been reported previously at the German Respiratory Society (DGP) conference 2014 in Bremen, Germany [45].

\section{Availability of data and materials}

Data are available from the corresponding author upon request.

\section{Authors' contributions}

Dr. MJR had full access to all of the data in the study and takes responsibility for the integrity of the data and the accuracy of the data analysis. He contributed as the principal investigator for this study to project oversight, organization, data collection, statistical analysis and writing of the manuscript. Dr. KM: contributed to the conceptual study design, to data collection, writing and critically reviewing of the manuscript and final approval of the version to be published. Dr. KT: contributed to the conceptual study design, to data collection, writing and critically reviewing of the manuscript and final approval of the version to be published. Mr. PS: contributed to the data collection, writing of the manuscript and final approval of the version to be published. Dr. WS: contributed to the data collection, writing of the manuscript and final approval of the version to be published. Dr. EM: contributed to the project organization, data collection, statistical analysis and writing of the manuscript. Dr. HAG: contributed to the conceptual study design, to data collection, writing and critically reviewing of the manuscript and final approval of the version to be published. Dr. HG: contributed to the conceptual study design, to data collection, writing and critically reviewing of the manuscript and final approval of the version to be published. All authors read and approved the final manuscript.

\section{Competing interests}

The authors declare that they have no competing interest.

\section{Author details}

${ }^{1}$ Department of Pneumology, Kerckhoff Heart, Rheuma and Thoracic Center, Bad Nauheim, Germany. ${ }^{2}$ Department of Internal Medicine,

Justus-Liebig-University Giessen, Universities of Giessen and Marburg Lung Center (UGMLC), Member of the German Center for Lung Research (DZL), Giessen, Germany. ${ }^{3}$ Department of Internal Medicine V, University of Munich, Comprehensive Pneumology Center, member of the German Center for Lung Research (DZL), Munich, Germany. ${ }^{4}$ Department of Thoracic Surgery, Kerckhoff Heart, Rheuma and Thoracic Center, Bad Nauheim, Germany.

${ }^{5}$ Department of Medicine, Imperial College London, London, UK.

Received: 5 March 2016 Accepted: 10 June 2016

Published online: 04 July 2016

\section{References}

1. Kim NH, Delcroix M, Jenkins DP, Channick R, Dartevelle P, Jansa P, Lang I, Madani MM, Ogino H, Pengo V, et al. Chronic thromboembolic pulmonary hypertension. J Am Coll Cardiol. 2013;62(25 Suppl):D92-9.

2. Delcroix M, Vonk Noordegraaf A, Fadel E, Lang I, Simonneau G, Naeije R. Vascular and right ventricular remodelling in chronic thromboembolic pulmonary hypertension. Eur Respir J. 2013;41(1):224-32.

3. Mayer E, Jenkins D, Lindner J, D'Armini A, Kloek J, Meyns B, Ilkjaer LB, Klepetko W, Delcroix M, Lang I, et al. Surgical management and outcome of patients with chronic thromboembolic pulmonary hypertension: results from an international prospective registry. J Thorac Cardiovasc Surg. 2011;141(3):702-10.

4. Corsico AG, D'Armini AM, Cerveri I, Klersy C, Ansaldo E, Niniano R, Gatto E, Monterosso C, Morsolini M, Nicolardi S, et al. Long-term outcome after pulmonary endarterectomy. Am J Respir Crit Care Med. 2008;178(4):419-24.

5. Kunihara T, Gerdts J, Groesdonk H, Sata F, Langer F, Tscholl D, Aicher D, Schafers $\mathrm{HJ}$. Predictors of postoperative outcome after pulmonary endarterectomy from a 14-year experience with 279 patients. Eur J Cardiothorac Surg. 2011;40(1):154-61.

6. Lankeit M, Dellas C, Panzenbock A, Skoro-Sajer N, Bonderman D, Olschewski M, Schafer K, Puls M, Konstantinides S, Lang IM. Heart-type fatty acidbinding protein for risk assessment of chronic thromboembolic pulmonary hypertension. Eur Respir J. 2008;31(5):1024-9.

7. Freed DH, Thomson BM, Tsui SS, Dunning JJ, Sheares KK, Pepke-Zaba J, Jenkins DP. Functional and haemodynamic outcome 1 year after pulmonary thromboendarterectomy. Eur I Cardiothorac Surg. 2008; 34(3):525-9. discussion 529-530.

8. Reesink HJ, van der Plas MN, Verhey NE, van Steenwijk RP, Kloek JJ, Bresser P. Six-minute walk distance as parameter of functional outcome after pulmonary endarterectomy for chronic thromboembolic pulmonary hypertension. J Thorac Cardiovasc Surg. 2007;133(2):510-6.

9. Suntharalingam J, Goldsmith K, Toshner M, Doughty N, Sheares KK, Hughes R, Jenkins D, Pepke-Zaba J. Role of NT-proBNP and 6MWD in chronic thromboembolic pulmonary hypertension. Respir Med. 2007; 101(11):2254-62.

10. Fox BD, Langleben D, Hirsch A, Boutet K, Shimony A. Step climbing capacity in patients with pulmonary hypertension. Clin Res Cardiol. 2013;102(1):51-61.

11. Casanova C, Cote C, Marin JM, Pinto-Plata V, de Torres JP, Aguirre-Jaime A, Vassaux C, Celli BR. Distance and oxygen desaturation during the 6-min walk test as predictors of long-term mortality in patients with COPD. Chest. 2008;134(4):746-52.

12. Lama VN, Flaherty KR, Toews GB, Colby TV, Travis WD, Long Q, Murray S, Kazerooni EA, Gross BH, Lynch JP 3rd, et al. Prognostic value of desaturation during a 6-minute walk test in idiopathic interstitial pneumonia. Am J Respir Crit Care Med. 2003;168(9):1084-90.

13. Provencher S, Chemla D, Herve P, Sitbon O, Humbert M, Simonneau G. Heart rate responses during the 6-minute walk test in pulmonary arterial hypertension. Eur Respir J. 2006;27(1):114-20. 
14. Lauer MS, Francis GS, Okin PM, Pashkow FJ, Snader CE, Marwick TH. Impaired chronotropic response to exercise stress testing as a predictor of mortality. JAMA. 1999;281(6):524-9.

15. Provencher $\mathrm{S}$, Mainguy V. Exercise Testing in Pulmonary Arterial Hypertension. Prog Respir Res. 2012;41:37-47.

16. Sun XG, Hansen JE, Oudiz RJ, Wasserman K. Exercise Pathophysiology in Patients With Primary Pulmonary Hypertension. Circulation. 2001;104(4):429-35.

17. Taboada D, Pepke-Zaba J, Jenkins DP, Berman M, Treacy CM, Cannon JE, Toshner M, Dunning JJ, Ng C, Tsui SS, et al. Outcome of pulmonary endarterectomy in symptomatic chronic thromboembolic disease. Eur Respir J. 2014;44(6):1635-45.

18. Authors/Task Force M, Galie N, Humbert M, Vachiery JL, Gibbs S, Lang I, Torbicki A, Simonneau G, Peacock A, Vonk Noordegraaf A et al.: 2015 ESC/ ERS Guidelines for the diagnosis and treatment of pulmonary hypertension: The Joint Task Force for the Diagnosis and Treatment of Pulmonary Hypertension of the European Society of Cardiology (ESC) and the European Respiratory Society (ERS)Endorsed by: Association for European Paediatric and Congenital Cardiology (AEPC), International Society for Heart and Lung Transplantation (ISHLT). Eur Heart J 2015. [Epub ahead of print].

19. Mayer E. Surgical and post-operative treatment of chronic thromboembolic pulmonary hypertension. Eur Respir Rev. 2010;19(115):64-7.

20. Freed DH, Thomson BM, Berman M, Tsui SS, Dunning J, Sheares KK, Pepke-Zaba J, Jenkins DP. Survival after pulmonary thromboendarterectomy: effect of residual pulmonary hypertension. J Thorac Cardiovasc Surg. 2011;141(2):383-7.

21. Thistlethwaite PA, Mo M, Madani MM, Deutsch R, Blanchard D, Kapelanski DP, Jamieson SW. Operative classification of thromboembolic disease determines outcome after pulmonary endarterectomy. J Thorac Cardiovasc Surg. 2002;124(6):1203-11.

22. Provencher $\mathrm{S}$, Herve P, Sitbon $\mathrm{O}$, Humbert M, Simonneau G, Chemla D. Changes in exercise haemodynamics during treatment in pulmonary arterial hypertension. Eur Respir J. 2008;32(2):393-8.

23. Azarbal B, Hayes SW, Lewin HC, Hachamovitch R, Cohen I, Berman DS. The incremental prognostic value of percentage of heart rate reserve achieved over myocardial perfusion single-photon emission computed tomography in the prediction of cardiac death and all-cause mortality: superiority over 85 \% of maximal age-predicted heart rate. J Am Coll Cardiol. 2004;44(2):423-30.

24. Andrianopoulos V, Franssen FM, Peeters JP, Ubachs TJ, Bukari H, Groenen M, Burtin C, Vogiatzis I, Wouters EF, Spruit MA. Exercise-induced oxygen desaturation in COPD patients without resting hypoxemia. Respir Physiol Neurobiol. 2014;190:40-6.

25. Laboratories ATSCoPSfCPF. ATS statement: guidelines for the six-minute walk test. Am J Respir Crit Care Med. 2002;166(1):111-7.

26. Janssens JP, de Muralt B, Titelion V. Management of dyspnea in severe chronic obstructive pulmonary disease. J Pain Symptom Manage. 2000;19(5):378-92.

27. Balady GJ, Arena R, Sietsema K, Myers J, Coke L, Fletcher GF, Forman D, Franklin B, Guazzi M, Gulati M, et al. Clinician's Guide to cardiopulmonary exercise testing in adults: a scientific statement from the American Heart Association. Circulation. 2010;122(2):191-225.

28. Scheidl SJ, Englisch C, Kovacs G, Reichenberger F, Schulz R, Breithecker A, Ghofrani HA, Seeger W, Olschewski H. Diagnosis of CTEPH versus IPAH using capillary to end-tidal carbon dioxide gradients. Eur Respir J. 2012; 39(1):119-24.

29. Rudski LG, Lai WW, Afilalo J, Hua L, Handschumacher MD, Chandrasekaran K Solomon SD, Louie EK, Schiller NB. Guidelines for the echocardiographic assessment of the right heart in adults: a report from the American Society of Echocardiography endorsed by the European Association of Echocardiography, a registered branch of the European Society of Cardiology, and the Canadian Society of Echocardiography. J Am Soc Echocardiogr. 2010;23(7):685-713. quiz 786-688.

30. van der Plas MN, Reesink HJ, Roos CM, van Steenwijk RP, Kloek JJ, Bresser P. Pulmonary endarterectomy improves dyspnea by the relief of dead space ventilation. Ann Thorac Surg. 2010;89(2):347-52.

31. Sato M, Ando M, Kaneko K, Higuchi Y, Kondo H, Akita K, Ishida M, Takagi $Y$. Respiratory and hemodynamic changes in patients with chronic thromboembolic pulmonary hypertension 1 year after pulmonary endarterectomy. Ann Vasc Dis. 2013;6(3):578-82

32. Pepke-Zaba J, Delcroix M, Lang I, Mayer E, Jansa P, Ambroz D, Treacy C, D'Armini AM, Morsolini M, Snijder R, et al. Chronic thromboembolic pulmonary hypertension (CTEPH): results from an international prospective registry. Circulation. 2011;124(18):1973-81.
33. Mauritz GJ, Vonk-Noordegraaf A, Kind T, Surie S, Kloek JJ, Bresser P, Saouti N Bosboom J, Westerhof N, Marcus JT. Pulmonary endarterectomy normalizes interventricular dyssynchrony and right ventricular systolic wall stress. J Cardiovasc Magn Reson. 2012;14:5.

34. Rahnavardi M, Yan TD, Cao C, Vallely MP, Bannon PG, Wilson MK. Pulmonary thromboendarterectomy for chronic thromboembolic pulmonary hypertension : a systematic review. Ann Thorac Cardiovasc Surg. 2011;17(5):435-45.

35. Hildenbrand FF, Bloch KE, Speich R, Ulrich S. Daytime measurements underestimate nocturnal oxygen desaturations in pulmonary arterial and chronic thromboembolic pulmonary hypertension. Respiration. 2012;84(6): 477-84.

36. McCabe C, Deboeck G, Harvey I, Ross RM, Gopalan D, Screaton N, PepkeZaba J. Inefficient exercise gas exchange identifies pulmonary hypertension in chronic thromboembolic obstruction following pulmonary embolism. Thromb Res. 2013:132(6):659-65.

37. Paciocco G, Martinez FJ, Bossone E, Pielsticker E, Gillespie B, Rubenfire M. Oxygen desaturation on the six-minute walk test and mortality in untreated primary pulmonary hypertension. Eur Respir J. 2001;17(4):647-52.

38. Domingo E, Grignola JC, Aguilar R, Arredondo C, Bouteldja N, Messeguer $\mathrm{ML}$, Roman A. Impairment of pulmonary vascular reserve and right ventricular systolic reserve in pulmonary arterial hypertension. BMC Pulm Med. 2014;14:69.

39. Vonk-Noordegraaf $A$, Westerhof $N$. Describing right ventricular function. Eur Respir J. 2013;41(6):1419-23.

40. Gerges C, Skoro-Sajer N, Lang IM. Right ventricle in acute and chronic pulmonary embolism (2013 Grover Conference series). Pulm Circ. 2014;4(3):378-86.

41. Reesink HJ, Marcus JT, Tulevski II, Jamieson S, Kloek JJ, Vonk Noordegraaf A, Bresser P. Reverse right ventricular remodeling after pulmonary endarterectomy in patients with chronic thromboembolic pulmonary hypertension: utility of magnetic resonance imaging to demonstrate restoration of the right ventricle. J Thorac Cardiovasc Surg. 2007;133(1):58-64.

42. Rolf A, Rixe J, Kim WK, Borgel J, Mollmann H, Nef HM, Liebetrau C, Kramm T, Guth S, Krombach GA, et al. Right ventricular adaptation to pulmonary pressure load in patients with chronic thromboembolic pulmonary hypertension before and after successful pulmonary endarterectomy - a cardiovascular magnetic resonance study. J Cardiovasc Magn Reson. 2014;16:96.

43. D'Armini AM, Zanotti G, Ghio S, Magrini G, Pozzi M, Scelsi L, Meloni G, Klersy C, Vigano M. Reverse right ventricular remodeling after pulmonary endarterectomy. J Thorac Cardiovasc Surg. 2007;133(1):162-8.

44. Deboeck G, Niset G, Vachiery JL, Moraine JJ, Naeije R. Physiological response to the six-minute walk test in pulmonary arterial hypertension. Eur Respir J. 2005;26(4):667-72

45. Stille P, Richter MJ, Wiedenroth CB, Zaatar M, Guth S, Voswinckel R: Hypoxämie im 6 Minuten Gehtest bei Patienten mit chronisch thromboembolischer pulmonaler Hypertonie. Pneumologie 2014, 68(S01):V218.

\section{Submit your next manuscript to BioMed Central and we will help you at every step:}

- We accept pre-submission inquiries

- Our selector tool helps you to find the most relevant journal

- We provide round the clock customer support

- Convenient online submission

- Thorough peer review

- Inclusion in PubMed and all major indexing services

- Maximum visibility for your research

Submit your manuscript at www.biomedcentral.com/submit 